Med Klin Intensivmed Notfmed 2012.

107:238-239

DOI 10.1007/s00063-011-0063-0

Online publiziert: 18. April 2012

(c) Springer-Verlag 2012

\title{
U. Janssens
}

Klinik für Innere Medizin und Intensivmedizin, St.-Antonius-Hospital, Eschweiler

\section{Intensivmedizin - Der Mensch im Mittelpunkt}

Sehr verehrte Kolleginnen und Kollegen!

Der Mensch im Mittelpunkt - unter diesem Leitthema findet die diesjährige gemeinsame Jahrestagung der Deutschen Gesellschaft für Internistische Intensivmedizin und Notfallmedizin (DGIIN) und der Österreichischen Gesellschaft für Internistische und Allgemeine Intensivmedizin und Notfallmedizin (ÖGIAIN) in Köln statt.

Die enormen technischen, diagnostischen und therapeutischen Erfolge der vergangenen Jahrzehnte haben zu einem fundamentalen demographischen Wandel mit immer älteren und multimorbiden Patienten nicht nur auf der Intensivstation geführt. Das Behandlungsteam auf der Intensivstation steht angesichts dieser Entwicklung vor immer größeren medizinischen Herausforderungen und den häufig damit verbundenen ethischen Problemen. Immer wieder sind im Grenzbereich zwischen Leben und Tod äußerst schwierige Entscheidungen zu treffen.

Aufgabe des Arztes ist es, unter Achtung des Selbstbestimmungsrechtes des Patienten, Leben zu erhalten, Gesundheit zu schützen und wiederherzustellen sowie Leiden zu lindern und Sterbenden bis zum Tod beizustehen. Die ärztliche Verpflichtung zur Lebenserhaltung besteht daher nicht unter allen Umständen. Es gibt Situatio$n e n$, in denen sonst angemessene Diagnostik- und Therapieverfahren nicht mehr angezeigt und Begrenzungen geboten sind. Dann tritt eine palliativmedizinische Versorgung in den Vordergrund.
Dieser Auszug aus den Grundsätzen der Bundesärztekammer zur ärztlichen Sterbebegleitung [1] gilt natürlich und insbesondere für den schwer kranken Intensivpatienten. Palliativmedizinische Konzepte müssen ihren Weg auch auf die Intensivstation finden, denn gerade dort werden sie dringend benötigt.

Eine regelrechte und adäquate Kommunikation im Team, zwischen Ärzten und Schwestern, oder mit Patienten und deren Angehörigen bleibt weiterhin eine zu wenig beachtete Aufgabe. Mängel in der Kommunikation tragen wesentlich zu Fehlern bei. Gerade auf Intensivstationen können Fehler die Prognose der Patienten entscheidend zu deren Nachteil verändern. Aus diesem Grund müssen wir uns viel aktiver und strukturierter als bisher um einer Verbesserung der Patientensicherheit kümmern. Ein modernes Risiko- und Qualitätsmanagement sollte auf jeder Intensivstation etabliert werden. Bezogen auf Kommunikation, Fehlermanagement und Qualitätsverbesserung sollten wir mehr als bisher auch auf die Erfahrungen und Konzepte des kommerziellen Luftverkehrs zurückgreifen.

Wir, die verantwortlichen Ärztinnen und Ärzte, müssen endlich anerkennen, dass die Intensivmedizin sich als High Reliability Organization (Hochzuverlässigkeitsorganisation) verstehen sollte. Wie in der Flugindustrie, bei Feuerwehren und Kernkraftwerken kommt es ganz besonders auf den professionellen Umgang mit unvorhergesehenen Ereignissen an. High Reliability Organizations [2] arbeiten nach 5 Prinzipien und - richten die Aufmerksamkeit eher auf ihre Fehler als auf ihre Erfolge, 
- schrecken vor grob vereinfachenden Interpretationen zurück,

- entwickeln ein feines Gespür für betriebliche Abläufe,

- streben nach Flexibilität und

- haben große Achtung vor fachlichem Wissen und Können und delegieren demzufolge die Entscheidungsverantwortung vor Ort.

Angesichts der zunehmenden Bedeutung der Intensiv- und Notfallmedizin im Gesundheitswesen und gleichzeitig eines wachsenden Mangels an qualifiziertem Fachpersonal (pflegerisch wie ärztlich) ist es unabdingbar, den Menschen in den Mittelpunkt unserer Bemühungen zu stellen. Eine qualitativ hochwertige Intensivmedizin darf sich nicht ausschließlich an einer rein medizinisch exzellenten Expertise ausrichten! Ethische Fragestellungen, der empathische Umgang mit Patienten, Angehörigen und Mitarbeitern, die Beachtung der Kommunikation, Stress und Burnout sowie die Etablierung eines robusten Fehlermanagements, aber auch ökonomische Analysen sind elementare Voraussetzungen einer modernen und vor allem zukunftsfähigen Intensivmedizin.

Wir werden ohne langfristige Personalentwicklungsmaßnahmen in den kommenden Jahren große Schwierigkeiten haben, Intensivstationen personell adäquat auszustatten. Die Fachgesellschaften sind hier gefordert, aber auch die Gesundheitspolitik. Neben einer kontinuierlichen Aus- und Weiterbildung im Schwerpunkt Intensivmedizin muss heutzutage jungen, engagierten Intensivmedizinerinnen und Intensivmedizinern auch eine berufliche Zukunft in der Intensivmedizin garantiert werden.

Ich bin als Kongresspräsident unserer diesjährigen Jahrestagung sehr froh, dass mir die Schriftleitung der Medizinische Klinik - Intensivmedizin und Notfallmedizin die Möglichkeit gegeben hat, einige dieser Aspekte in einem kongressbegleitenden Themenheft aufzugreifen. Dabei möchte ich den Autoren ausdrücklich für ihre hervorragenden Beiträge danken! Angesichts der hohen Arbeitsbelastung ist es keine Selbstverständlich- keit, Übersichten von dieser Qualität zu verfassen.

Ich darf Ihnen viel Freude beim Lesen dieser Ausgabe wünschen.

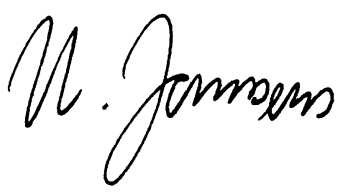

Prof. Dr. Uwe Janssens

\section{Korrespondenzadresse}

Prof. Dr. U. Janssens
Klinik für Innere Medizin
und Intensivmedizin,
St.-Antonius-Hospital
Dechant-Deckers-Str. 8,
52249 Eschweiler
uwe.janssens@
sah-eschweiler.de

\section{Literatur}

1. Bundesärztekammer (2011) Grundsätze der Bundesärztekammer zur Sterbebegleitung. Dtsch Arztebl 108:A346-A348

2. Sutcliffe KM (2011) High reliability organizations (HROs). Best Pract Res Clin Anaesthesiol 25:133144

\section{Risikomanagement in der Akutmedizin}

Im Mittelpunkt jeder qualitätsorientierten Gesundheitsversorgung steht die Sicherheit des Patienten. Gerade die Notfall- und die Akutmedizin ist ein Hochrisikoumfeld, in dem präventivem und systemorientierten Risikomanagement eine zentrale Bedeutung zukommt. Wichtig ist dabei, Dokumentation und Analyse im Risikoassessment gut in den notfallmedizinischen Alltag zu integrieren.

In der Ausgabe
Botfall+
Bettungsmedizin
+ Rettungsmedizin
gibt das Leit-
thema „Risiko-
management in
der Akutmedizin“
einen Überblick
über aktuelle
wissenschaftliche

Erkenntnisse und erfolgreiche Anwendungsstrategien.

Die Schwerpunkte des Leitthemenheftes sind:

-Risikomanagement und Human Factor in der Akutmedizin -Strukturiertes klinisches Risikomanagement in einer Akutklinik -Critical-Incident-Reporting-System (CIRS)

-Risikoassessment zur Schadenprävention in der Akutmedizin -Medikamentengabe durch nichtärztliches Rettungsfachpersonal

Bestellen Sie diese Ausgabe zum Preis von $35,-€$ bei

Springer Customer Service Center Kundenservice Zeitschriften

Haberstr. 7

69126 Heidelberg

Tel.: +49 6221-345-4303

Fax: +49 6221-345-4229

E-Mail: leserservice@springer.com

PS Vieles mehr rund um Ihr Fachgebiet finden Sie auf www.springermedizin.de 